\title{
Numerical Method of Dynamic Buckling of Bar Impacted by a Rigid Body
}

\author{
Guangyuan Zhang ${ }^{1}$, Zhijun $\mathrm{Han}^{2, *}$ and Guoyun $\mathrm{Lu}^{3}$ \\ ${ }^{1}$ College of Mechanics, Taiyuan University of Technology, Taiyuan 030600, China \\ ${ }^{2}$ College of Mechanics, Taiyuan University of Technology, Taiyuan 030600, China \\ ${ }^{3}$ College of Architecture and Civil Engineering, Taiyuan University of Technology, Taiyuan 030024, China \\ ${ }^{*}$ Corresponding author
}

\begin{abstract}
The issues of dynamic buckling of elastic bar impacted by a rigid body before and after the stress wave reflection were studied by considering the stress wave effect. Based on the Hamiltonian principle, the dynamic buckling control equations and constraint conditions at the wavefront were derived in the case of axial inertia and transverse inertia. All the equations and conditions should be dimensionless, and the governing equations were resolved by the difference method and the boundary condition and continuity condition were solved by forward and backward difference method. The buckling waveforms of the elastic rods under different impact speed, impact mass and section size were obtained. The influence of the impact speed, the impact mass and the cross-sectional dimension on the dynamic buckling of the elastic rods after the stress wave reflection were analyzed. The results show that the critical velocity decreases with the increase of impact mass and critical length, and increases with the increase of inertia radius. The higher modes would be excited with the higher impact velocity easily. The number of buckling modes, wave crests and trough valleys increased with the increase of the propagation distance, indicating that the stress wave effect has a great influence on dynamic buckling.
\end{abstract}

Keywords—rigid body; dynamic buckling; critical speed; stress wave; finite difference method

\section{INTRODUCTION}

Straight rod has been widely used in many industrial fields such as aerospace, construction engineering and mechanical engineering and national defense construction as the most commonly used basic component in engineering structure. The dynamic buckling problem caused by its impact and so on has always been valued by people in the study of structural stability $^{[1 \sim 2]}$. However, there are few studies on the dynamic buckling problem of the rigid mass axially impacting the elastic rod due to the complexity of axial stress waves. AriGur $\mathrm{J}^{[3]}$ studied the buckling problem of the straight bar impacted by rigid mass without using the stress wave effect by the difference method. Han Zhijun ${ }^{[4]}$ studied the dynamic buckling problem of the rigid rod with axial impact, analyzed the propagation of the stress wave in the straight rod by characteristic line theory and gave the problem series solution. Heidarpour, $\mathrm{A}^{[5]}$ proposed a new semi-analytical non-discrete numerical method for the nonlinear dynamic analysis of steel beam-column elements under explosive loads. The results obtained are basically consistent with those obtained by finite element simulation using ABAQUS. Phani ${ }^{[6]}$ studied the elastic dynamic buckling problem of the Ouranoulouli beam under axial load and compared the numerical results with the dynamic response of a large number of finite element models to test the validity of the dynamic buckling formula. D. KAGAGIOZOVA ${ }^{[7 \sim 8]}$ studied the buckling of elastic-plastic rod and shell under axial load considering the influence of geometry and material properties and the loading conditions. At the same time, the propagation of stress waves in fixed foam blocks impacted by small masses is studied to investigate the compaction mechanism before and after stress wave reflection. $\mathrm{MaHao}^{[9]}$ used the finite difference method to analyze the dynamic buckling problem of the elastic rod under step load.

To sum up, when considering the stress wave effect, especially the stress wave is reflected, the buckling of the mass impact rod is more complicated and it is difficult to obtain the analytic expression. Thus, the numerical solution becomes a method to discuss such problems. Based on this, this paper studied the buckling problem of mass axial impact elastic rod. Based on the Hamiltonian principle, the dynamic buckling control equations and constraint conditions at the wave-front were derived in the case of axial inertia and transverse inertia. All the equations and conditions should be dimensionless, and the governing equations were resolved by the difference method. The influence of the impact speed, the impact mass and the cross-sectional dimension on the dynamic buckling of the elastic rods after the stress wave reflection were discussed.

\section{GOVERNING EQUATION OF THE DYNAMIC BUCKLING}

Figure I is an ideal elastic straight rod of length $L$ with one end clamped and one end clamped. It is impacted by a rigid mass of velocity $V_{0}$ and mass $M$.

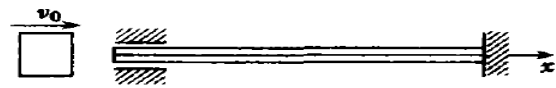

FIGURE I. ELASTIC BAR IMPACTED BY A RIGID BODY

The propagation process of Stress wave in the rod can be expressed as ${ }^{[4]}$ :

(1). $0 \leq t \leq L / C$ 


$$
\begin{aligned}
& \sigma(x, t)= \begin{cases}-\rho c V_{0} e^{-\rho A c t / M+\rho A x / M}, & 0 \leq x \leq c t \\
0 & c t\end{cases} \\
& c t \leq x \leq L
\end{aligned}
$$

(2). $L / c \leq t \leq 2 L / c$

$$
\sigma(x, t)= \begin{cases}-\rho c V_{0} e^{-\rho A c t / M+\rho A x / M}, & 0 \leq x \leq 2 L-c t \\ -2 \rho c V_{0} e^{-\rho A c t / M-\rho A x / M+2 \rho A L / M}, & 2 L-c t \leq x \leq L\end{cases}
$$

When the stress wave propagates in the rod to $l$, the rod suddenly buckles and the bending of the rod conforms to the assumption of flat section so that the rod can only move in the main section (x,y). All the stress and displacement are independent of $\mathrm{z}$, and any point of the z-direction displacement are zero. The displacements in $\mathrm{x}$ and $\mathrm{y}$ directions are $\mathrm{u}$ and $\mathrm{w}$ respectively. Strain at any point of the rod cross-section can be expressed as:

$$
\varepsilon=\frac{\partial u}{\partial x}+\frac{1}{2}\left(\frac{\partial w}{\partial x}\right)^{2}-z \frac{\partial^{2} w}{\partial x^{2}}=u^{\prime}+\frac{1}{2} w^{\prime 2}-z w^{\prime \prime}
$$

Kinetic energy, strain energy and external force of rod:

$$
\begin{gathered}
T=\frac{1}{2} \rho b \int_{0}^{l} \int_{-h / 2}^{h / 2}\left(\dot{w}^{2}+\dot{u}^{2}\right) d z d x \\
U=\frac{1}{2} b \int_{0}^{l} \int_{-h / 2}^{h / 2} \sigma \varepsilon d z d x \\
W=P(t) u(0, t)
\end{gathered}
$$

Apply Hamilton principle:

$$
\delta \int_{t_{1}}^{t_{2}}(T-U+W) d t=0
$$

Substituting equations (4) to (6) into (7) gives two control equations and a set of constraints at the wave-front are:

$$
\begin{gathered}
N^{\prime}+\rho A \ddot{u}=0 \\
E I w^{\prime \prime \prime}+\left(N w^{\prime}\right)^{\prime}+\rho A \ddot{w}=0 \\
w(l)=w^{\prime}(l)=0
\end{gathered}
$$

\section{A. Before the Stress Wave Reflection}

When Stress wave reflected before that is $0 \leq t \leq L / c$ and the distance from the impact side of the wave-front is $l_{1}\left(l_{1}=c t\right)$, the dynamic buckling of rod happens.

If ignoring the shear deformation, axial inertia and rotational inertia effect, the equation of dynamic buckling can be obtained from Eq. (9) is:

$$
E I w^{\prime \prime \prime \prime}+\left(N w^{\prime}\right)^{\prime}+\rho A \ddot{w}=0
$$

Where $I$ is the moment of inertia of the cross section and $w$ is the lateral displacement; $L_{c}=c_{e} t_{c r}, i=\sqrt{I / A}$, taking the following non-dimensional quantities: $\bar{w}=w / i, \bar{x}=x / L_{c}$, $\bar{t}=t / t_{c r}, \bar{N}=N / E A$.

The dimensionless form of Eq. (11) is:

$$
\frac{i^{2}}{L_{c}^{2}} \bar{w}^{\prime \prime \prime}+\bar{N}^{\prime} \cdot \bar{w}^{\prime}+\bar{N} \cdot \bar{w}^{\prime \prime}=-\stackrel{\bar{w}}{ }
$$

Equation (12) satisfies the following boundary conditions and initial conditions:

Impact end for the folder, Wave-front to meet the branch support:

$$
\begin{array}{ll}
\bar{w}(0, \bar{t})=0, & \bar{w}^{\prime}(0, \bar{t})=0 \\
\bar{w}\left(\bar{l}_{1}, \bar{t}\right)=0, & \bar{w}^{\prime}\left(\bar{l}_{1}, \bar{t}\right)=0
\end{array}
$$

The initial lateral displacement ${ }^{[4]}$ is:

$$
\bar{w}_{0}=c \cdot[3 \sin (\pi \bar{x})-\sin (3 \pi \bar{x})]
$$

Where $c$ is a tiny parameter.

The initial condition of lateral displacement $\bar{w}$ is:

$$
\bar{w}=\bar{w}_{0}, \dot{\bar{w}}=\dot{\bar{w}}_{0} \quad(0 \leq \bar{x} \leq 1)
$$

$$
\bar{w}=0, \dot{\bar{w}}=0 \quad\left(1 \leq \bar{x} \leq L / L_{c}\right)
$$




\section{B. After the Stress Wave Reflection}

When Stress wave reflected after that is $L / c \leq t \leq 2 L / C$ and the distance from the impact side of the wave-front is $l_{2}\left(l_{2}=2 L-c t\right)$, the dynamic buckling of rod happens.

If ignoring the shear deformation, axial inertia and rotational inertia effect, the equation of dynamic buckling can be obtained from Eq. (9) is:

$$
E_{i} I w_{i}^{\prime \prime \prime}+\left(N_{i} w_{i}^{\prime}\right)^{\prime}+\rho A \ddot{w}_{i}=0
$$

Where $i=1$, that is when $0 \leq x \leq l_{2}, w_{1}$ is the disturbance solution. Where $i=2$, that is when $l_{2} \leq x \leq L, w_{2}$ is the buckling solution.

The dimensionless form of Eq. (18) is:

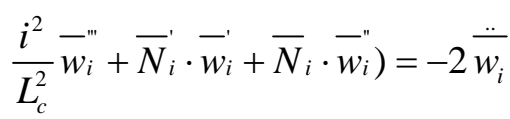

Equation (19) satisfies the following boundary conditions, continuity conditions and initial conditions at the wave-front:

Impact end for the folder, the other end of the solid support:

$$
\begin{array}{ll}
\bar{w}_{1}(0, \bar{t})=0, & \bar{w}_{1}^{\prime}(0, \bar{t})=0 \\
\bar{w}_{2}\left(\bar{l}_{2}, \bar{t}\right)=0, & \bar{w}_{2}^{\prime}\left(\bar{l}_{2}, \bar{t}\right)=0
\end{array}
$$

Displacement, corner, moment and shear satisfy the continuity condition at the wave-front:

$$
\begin{aligned}
& \bar{w}_{1}\left(\bar{l}_{2}, \bar{t}\right)=\bar{w}_{2}\left(\bar{l}_{2}, \bar{t}\right) \\
& \bar{w}_{1}^{\prime}\left(\bar{l}_{2}, \bar{t}\right)=\bar{w}_{2}^{\prime}\left(\bar{l}_{2}, \bar{t}\right) \\
& \bar{w}_{1}^{\prime \prime}\left(\bar{l}_{2}, \bar{t}\right)=\bar{w}_{2}^{\prime \prime}\left(\bar{l}_{2}, \bar{t}\right) \\
& \frac{i^{2} \bar{w}_{1}\left(\bar{l}_{2}, \bar{t}\right)+\bar{N}_{1} \cdot \bar{w}_{1}^{\prime}\left(\bar{l}_{2}, \bar{t}\right)}{L_{c}^{2}}=\frac{i^{2}}{L_{c}^{2}} \bar{w}_{2}^{\prime \prime \prime}\left(\bar{l}_{2}, \bar{t}\right)+\bar{N}_{2} \cdot \bar{w}_{2}^{\prime}\left(\bar{l}_{2}, \bar{t}\right)
\end{aligned}
$$

The initial lateral displacement ${ }^{[4]}$ is:

$$
\begin{gathered}
\bar{w}_{0}=c \cdot[3 \sin (\pi \bar{x})-\sin (3 \pi \bar{x})] \\
\bar{w}_{01}=c_{0} \cdot\left[e^{\pi \bar{x}} \sin (3 \pi \bar{x})-e^{-\pi \bar{x}} \sin (3 \pi \bar{x})\right]
\end{gathered}
$$

Where $c$ and $c_{0}$ are tiny parameters. $c=0.01 \mathrm{~h} / \mathrm{i}, c_{0}=0.01 \mathrm{c}$.

The initial condition of lateral displacement $\bar{w}$ is:

$$
\bar{w}_{1}=\bar{w}_{01}, \dot{\bar{w}}_{1}=\dot{\bar{w}}_{01} \quad\left(0 \leq \bar{x} \leq l_{2} / L_{c}\right)
$$

$$
\bar{w}_{2}=\bar{w}_{0}, \dot{\bar{w}}_{2}=\dot{\bar{w}}_{0} \quad\left(l_{2} / L_{c} \leq \bar{x} \leq L / L_{c}\right)
$$

\section{FINITE DifFERENCE SCHEME OF CONTROL EQUATION}

The finite difference method is used to solve the governing equations. The left and right boundary conditions are resolved by using the forward and backward difference methods respectively. The wave-front continuity conditions are resolved by using backward and forward difference methods respectively.

In the above formula, the subscript $i$ indicates the space point, the subscript $j$ indicates the time point, and the difference to the time derivative is the same as above, $\Delta \bar{x}, \Delta \bar{t}$ are the dimensionless quantities of displacement and time step respectively. When $\Delta \bar{x}=\Delta \bar{t}=0.025$, a stable differential result is obtained.

The above difference form is substituted into the control equation, and the difference form of the governing equation is obtained by the solution:

$$
\bar{w}_{j+1}=-(\Delta \bar{t})^{2}\left(\frac{i^{2}}{L_{c}^{2}} \bar{w}_{1}^{\prime \prime}-\bar{N} \cdot \bar{w}_{i}^{\prime}-\bar{N} \cdot \bar{w}_{i}^{\prime \prime}\right)+2 \bar{w}_{j}-\bar{w}_{j-1}
$$

\section{NUMERICAL ANALYSIS}

Programming equations (30) by using Matlab. Taking the rod cross-sectional size of $b \times h=5.85 \times 10.1 \mathrm{~mm}^{2}$, rod length $L=1 \mathrm{~m}, \rho=7.8 \times 10^{-3} \mathrm{~g} / \mathrm{mm}^{2}, E=200 \mathrm{Gpa}$, $c=0.01 h / i, c_{0}=0.01 c$, number of modalities $m$, impact quality $M$, critical speed $v_{0}$, inertial radius $i$, critical length $L_{c}$, the results obtained by the $w=\bar{w} \times i$ can be the actual longitudinal displacement, the results are as follows: 
A. Before the Stress wave reflection

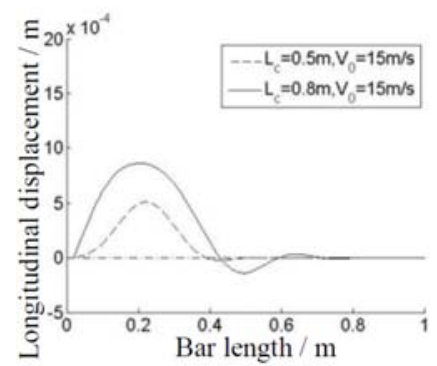

(a)

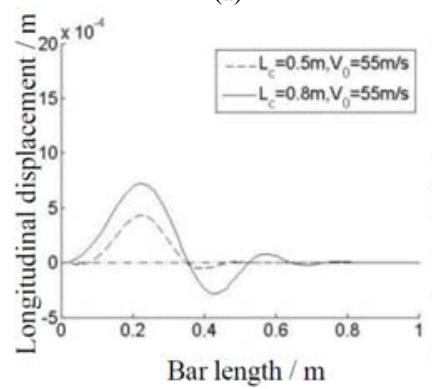

(c)

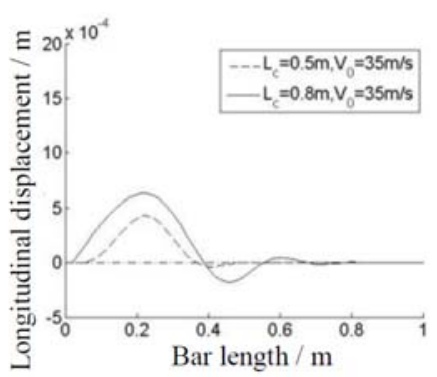

(b)

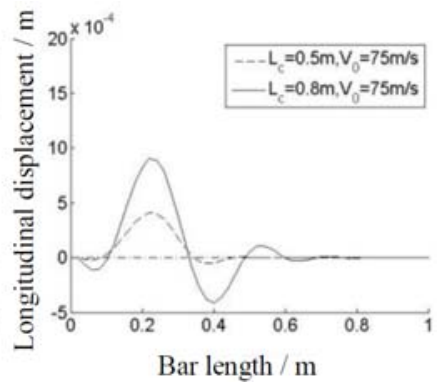

(d)

FIGURE II. THE OSCILLOGRAM OF BUCKLING OF BAR UNDER DIFFERENT IMPACT VELOCITY

TABLE I. THE KEY VALUES OF OSCILLOGRAM UNDER DIFFERENT IMPACT VELOCITY

\begin{tabular}{|c|c|c|c|c|}
\hline Waveform number & a & b & c & d \\
\hline Critical speed (m/s) & 0.5 & 0.5 & 0.5 & 0.5 \\
\hline Critical length $(\mathbf{m})$ & \multicolumn{5}{|c|}{0.5} \\
\hline Peak value $\left(\times \mathbf{1 0}^{-4} \mathbf{m}\right)$ & 5.164 & 4.313 & 4.327 & 4.357 \\
\hline Valley value $\left(\times \mathbf{1 0}^{-4} \mathbf{m}\right)$ & -4.57 & -3.545 & -5.534 & -4.839 \\
\hline Critical length $(\mathbf{m})$ & \multicolumn{5}{|c|}{0.8} \\
\hline Peak value $\left(\times \mathbf{1 0}^{-4} \mathbf{m}\right)$ & 8.563 & 6.309 & 7.221 & 9.055 \\
\hline Valley value $\left(\times \mathbf{1 0}^{-4} \mathbf{m}\right)$ & -1.404 & -1.77 & -2.818 & -4.138 \\
\hline
\end{tabular}

Figure II shows the waveforms of straight bar buckling when $M$ and $i$ are constant, $L_{c}$ are $0.5 \mathrm{~m}$ and $0.8 \mathrm{~m}$ respectively and $i$ is not equal. Table I shows the key figures corresponding to the waveform. It shows that when $L_{c}$ is different and $v_{0}$ is the same, the half-wave length, the number of buckling modes, the peak value and the valley value of the waveform increase as $L_{c}$ increases(Figure a). when $v_{0}$ is different and $L_{c}$ is the same, the half-wave length decreases as $v_{0}$ increases, the number of buckling modes, the peak value and the valley value of the waveform increase as $v_{0}$ increases. It shows again that the stress wave effect have a greater impact on dynamic buckling.

B. After the Stress wave reflection

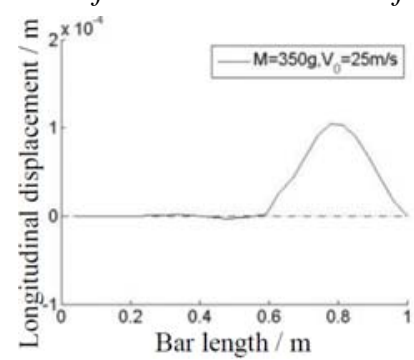

(a)

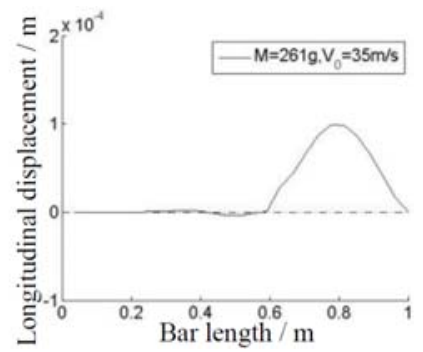

(b)

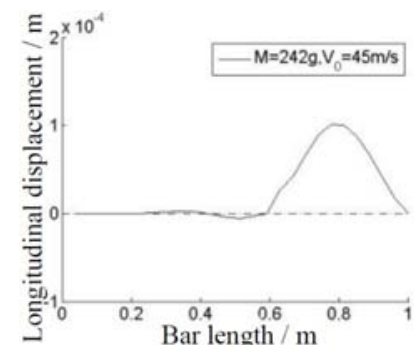

(c)

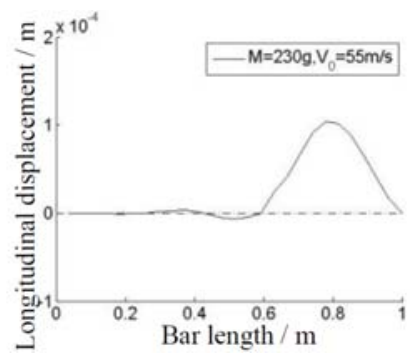

(d)
FIGURE III. THE OSCILLOGRAM OF BUCKLING OF BAR UNDER DIFFERENT IMPACT MASS

TABLE II. THE KEY VALUES OF OSCILLOGRAM UNDER DIFFERENT IMPACT MASSES

\begin{tabular}{|c|c|c|c|c|}
\hline Waveform number & a & b & c & d \\
\hline Critical length (m) & 0.4 & 0.4 & 0.4 & 0.4 \\
\hline Critical speed (m/s) & 25 & 35 & 45 & 55 \\
\hline Impact quality (g) & 350 & 261 & 242 & 230 \\
\hline Peak value $\left(\times \mathbf{1 0}^{-4} \mathbf{m}\right)$ & 1.031 & 1.001 & 1.015 & 1.038 \\
\hline
\end{tabular}

Figure III shows the waveforms of straight bar buckling when $L_{c}, m$ and $i$ are constant and $M$ is not equal. Table II shows the key figures corresponding to the waveform. It shows that the smaller $M$ is, the larger $v_{0}$ is, that is $v_{0}$ decreases with the increase of $\mathrm{M}$. The half wavelength of the waveform is the stress wave propagation distance that is $L_{c}$, while the peak value is substantially unchanged. The disturbance solution is much smaller than the buckling solution, and its influence can be neglected. It shows that the reflected wave-front disturbance has negligible effect on dynamic buckling.

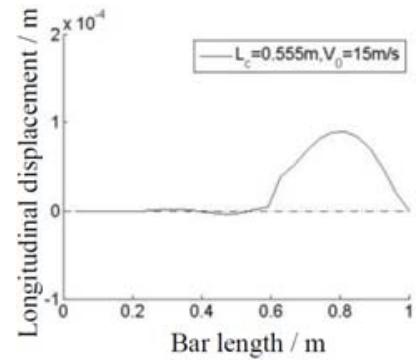

(a)

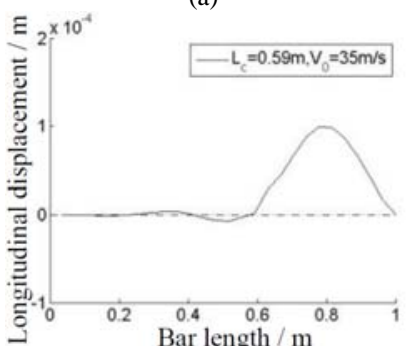

(c)

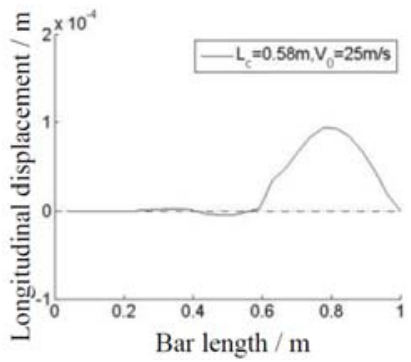

(b)

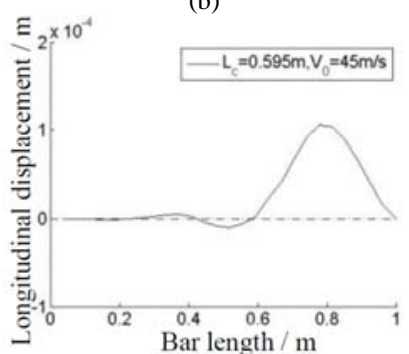

(d)
FIGURE IV. THE OSCILLOGRAM OF BUCKLING OF BAR UNDER DIFFERENT CRITICAL LENGTH 
TABLE III. THE KEY VALUES OF OSCILLOGRAM UNDER DIFFERENT CRITICAL LENGTH

\begin{tabular}{|c|c|c|c|c|}
\hline Waveform number & a & b & c & d \\
\hline Critical length (m) & $1-0.555$ & $1-0.58$ & $1-0.59$ & $1-0.595$ \\
\hline Critical speed (m/s) & 15 & 25 & 35 & 45 \\
\hline Peak value $\mathbf{( \times \mathbf { 1 0 } ^ { - 4 } \mathbf { m } )}$ & 0.888 & 0.934 & 0.992 & 1.056 \\
\hline
\end{tabular}

Figure IV shows the waveforms of straight bar buckling when $M, m$ and $i$ are constant and $L_{c}$ is not equal. Table III shows the key figures corresponding to the waveform. It shows that the smaller $L_{c}$ is, the larger $v_{0}$ is, that is $v_{0}$ decreases with the increase of $L_{c}$ and the critical length of the stress wave has a significant effect on the critical speed, which has a significant effect on dynamic buckling. The half-wave of the waveforms decrease and peak value increase gradually. It shows again that the stress wave effect has a significant effect on the buckling. The amplitude of perturbation solution also increases slightly with the increase of velocity, and is much smaller than buckling solution. The results show that the reflected wavefront disturbance has negligible effect on the dynamic buckling.

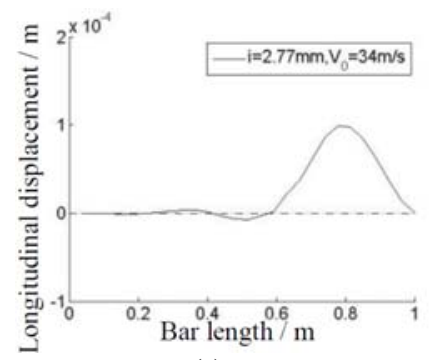

(a)

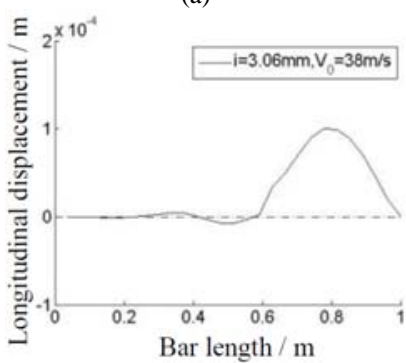

(c)

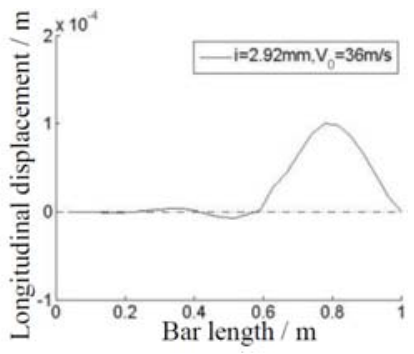

(b)

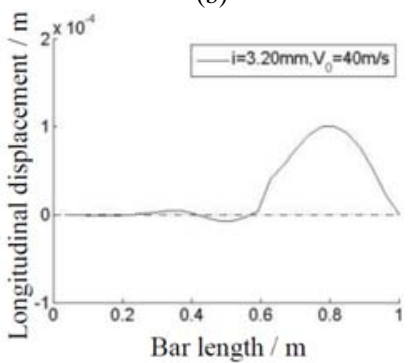

(d)
FIGURE V. THE OSCILLOGRAM OF BUCKLING OF BAR UNDER DIFFERENT INERTIAL RADIUS

TABLE IV. THE KEY VALUES OF OSCILLOGRAM UNDER DIFFERENT INERTIAL RADIUS

\begin{tabular}{|c|c|c|c|c|}
\hline Waveform number & a & b & c & d \\
\hline Critical length (m) & 0.4 & 0.4 & 0.4 & 0.4 \\
\hline Critical speed (m/s) & 34 & 36 & 38 & 40 \\
\hline Inertial radius (mm) & 2.77 & 2.92 & 3.06 & 3.20 \\
\hline Peak value $\left(\times \mathbf{1 0}^{-4} \mathbf{m}\right)$ & 0.991 & 0.998 & 0.999 & 1.002 \\
\hline
\end{tabular}

Figure $\mathrm{V}$ shows the waveforms of straight bar buckling when $m$ and $M$ are constant and $i$ is not equal. Table IV shows the key figures corresponding to the waveform. It shows that the larger $i$ is, the larger $v_{0}$ is, that is $v_{0}$ decreases with the increase of $i$. Half-wave length of waveforms are constant and peak value amplitude increase is smaller. It shows that the radius of inertia also have effect on the buckling. With the increase of the radius of inertia, the amplitude of the perturbed solution increases less and is much smaller than buckling solution. The results show that the reflected wave-front disturbance has negligible effect on the dynamic buckling. As can be seen from Fig. III to Fig. V, as the velocity increases, the buckling develops gradually and the wave-front disturbance also slightly increases, but does not affect the occurrence of buckling.

\section{CONCLUSION}

The following conclusions can be drawn from theoretical analysis and numerical calculation:

i) Based on the Hamiltonian principle, the dynamic buckling control equations and constraint conditions at the wave-front were derived in the case of axial inertia and transverse inertia.

ii) Dynamic control equations should be dimensionless. The left and right boundary conditions are resolved by using the forward and backward difference methods respectively. The wave-front continuity conditions are resolved by using backward and forward difference methods respectively. The finite difference method is used to solve the governing equations and the difference form of the governing equation is obtained by the solution.

iii) Using MATLAB to calculate the difference scheme. The influences of impact quality, critical length and inertia radius on the critical speed are discussed. The number of buckling modes, peak value and valley value increase with the propagation distance increasing. It shows that the stress wave effect has a great influence on dynamic buckling.

The critical velocity of dynamic buckling of elastic rod impacted by a mass decreases with the increase of impact mass and critical length, and increases with the increase of inertial radius after axial stress wave reflection .The reflected wavefront disturbance has negligible effect on the dynamic buckling.

\section{ACKNOWLEDGMENT}

This research was financially supported by the National Science Foundation of China (No.11372209)

\section{REFERENCES}

[1] Frikha, Ahmed, F. Treyssède, and P. Cartraud. "Effect of axial load on the propagation of elastic waves in helical beams." Wave Motion 48.1(2011),pp.83-92.

[2] Karagiozova, D. "Dynamic buckling of elastic-plastic square tubes under axial impact-I: stress wave propagation phenomenon. " International Journal of Impact Engineering 30. 2(2004),pp.143-166.

[3] Ari-Gur, J., T. Weller, and J. Singer. "Experimental and theoretical studies of columns under axial impact $i t$." International Journal of Solids \& Structures18.7(1982),pp.619-641.

[4] Han, et al. "THE DYNAMIC BUCKLING OF ELASTIC-PLASTIC COLUMN SUBJECTED TO AXIAL IMPACT BY A RIGID BODY." Acta Mechanica Solida Sinica.18. 3(2005),pp.256-264.

[5] Heidarpour, A., and M. A. Bradford. "Beam-column element for nonlinear dynamic analysis of steel members subjected to blast loading." Engineering Structures 33.4(2011),pp.1259-1266.

[6] Motamarri, Phani, and S. Suryanarayan. "Unified analytical solution for dynamic elastic buckling of beams for various boundary conditions and loading rates." International Journal of Mechanical Sciences56.1(2012),pp.60-69. 
[7] Karagiozova, D, and M. Alves. "Dynamic Elastic-Plastic Buckling of Structural Elements: A Review." Applied Mechanics Reviews. 61.4(2008),pp.967-973.

[8] Karagiozova D, Alves M. "Primary and reflected compaction waves in a foam rod due to an axial impact by a small mass". Latin American Journal of Solids \& Structures, 12(5),2015,pp.905-924.

[9] Hao Ma, Qiang Yang, Zhi-jun Han, et al. "Dynamic buckling of elastic bar under axial impact based on finite difference method. " International Conference an Advanced Material Science and Engineering. 2016,pp. 164-169. 